\title{
Microdeletions excluding YWHAE and PAFAH1B1 cause a unique leukoencephalopathy: further delineation of the 17p13.3 microdeletion spectrum
}

\author{
Lisa T. Emrick, $\mathrm{MD}^{1,2,3}$, Jill A. Rosenfeld, $\mathrm{MS}^{3}$, Seema R. Lalani, $\mathrm{MD}^{2,3,4}$, Mahim Jain, MD PhD ${ }^{3,10}$, \\ Nilesh K. Desai, MD ${ }^{5}$, Austin Larson, MD ${ }^{6}$, Kimberly Kripps, MD ${ }^{6}$, Adeline Vanderver, MD”, \\ Ryan J. Taft, $\mathrm{PhD}^{8}$, Krista Bluske, $\mathrm{PhD}^{8}$, Denise Perry, $\mathrm{MS}^{8}$, Honey Nagakura, $\mathrm{MS}^{9,11}$, \\ LaDonna L. Immken, $\mathrm{MD}^{9}$, Lindsay C. Burrage, MD PhD ${ }^{2,3}$, Carlos A. Bacino, MD ${ }^{2,3,4}$, \\ John W. Belmont, MD PhD ${ }^{3,12}$, Undiagnosed Diseases Network and Brendan Lee, MD PhD ${ }^{2,3}$
}

\begin{abstract}
Purpose: Brain malformations caused by 17 p13.3 deletions include lissencephaly with deletions of the larger Miller-Dieker syndrome region or smaller deletions of only PAFAH1B1, white matter changes, and a distinct syndrome due to deletions including YWHAE and CRK but sparing PAFAH1B1. We sought to understand the significance of $17 \mathrm{p} 13.3$ deletions between the $Y W H A E / C R K$ and PAFAH1B1 loci.
\end{abstract}

Methods: We analyzed the clinical features of six individuals from five families with 17p13.3 deletions between and not including YWHAE/ $C R K$ and $P A F A H 1 B 1$ identified among individuals undergoing clinical chromosomal microarray testing or research genome sequencing.

Results: Five individuals from four families had multifocal white matter lesions while a sixth had a normal magnetic resonance image. A combination of our individuals and a review of those in the literature with white matter changes and deletions in this chromosomal region narrows the overlapping region for this brain phenotype to $\sim 345 \mathrm{~kb}$, including 11 RefSeq genes, with RTN4RL1 haploinsufficiency as the best candidate for causing this phenotype.

Conclusion: While previous literature has hypothesized dysmorphic features and white matter changes related to YWHAE, our cohort contributes evidence to the presence of additional genetic changes within 17 p13.3 required for proper brain development.

Genetics in Medicine (2019) 21:1652-1656; https://doi.org/10.1038/s41436018-0358-0

Keywords: 17p13.3 microdeletion; leukoencephalopathy; chromosomal microarray; white matter

\section{INTRODUCTION}

There is a spectrum of microdeletion syndromes associated with 17p13.3 deletions. Miller-Dieker syndrome (OMIM 247200), associated with severe lissencephaly, dysmorphic features and multiple congenital anomalies, occurs when a deletion includes YWHAE and PAFAH1B1 (LIS1). Isolated lissencephaly sequence (OMIM 607432), with primarily only lissencephaly occurs with smaller deletions involving PAFAH1B1 but not $Y W H A E .{ }^{1}$ Individuals with smaller deletions including YWHAE and CRK but sparing PAFAH1B1 have growth restriction, cognitive impairment, dysmorphic features, and various brain abnormalities. $^{2-4}$ White matter abnormalities have been reported in some individuals with $17 \mathrm{p} 13.3$ microdeletions, and previous literature has hypothesized that this may be related to YWHAE haploinsufficiency. ${ }^{2-5}$ We report a cohort of children with primarily white matter changes on brain MRI but no significant cognitive impairment. All have small interstitial 17p13.3 microdeletions proximal to $C R K$, which has been hypothesized to be associated with short stature, ${ }^{2,3}$ and distal to PAFAH1B1, associated with lissencephaly. ${ }^{1}$

\section{MATERIALS AND METHODS}

\section{Subject ascertainment}

This study was approved by the Baylor College of Medicine Institutional Review Board for Human Subjects Research. Individual 1 enrolled in the Undiagnosed Diseases Network

\footnotetext{
${ }^{1}$ Department of Neurology, Baylor College of Medicine, Houston, TX, USA; ${ }^{2}$ Department of Pediatrics, Baylor College of Medicine, Houston, TX, USA; ${ }^{3}$ Department of Molecular \& Human Genetics, Baylor College of Medicine, Houston, TX, USA; ${ }^{4}$ Baylor Genetics, Houston, TX, USA; ${ }^{5}$ Department of Radiology, Baylor College of Medicine, Houston, TX, USA; ${ }^{6}$ Section of Genetics, Department of Pediatrics, University of Colorado School of Medicine, Aurora, CO, USA; 7 Division of Neurology, Children's Hospital of Philadelphia, Philadelphia, PA, USA; ${ }^{8}$ Illumina, San Diego, CA, USA; ${ }^{9}$ Dell Children's Medical Center, Austin, TX, USA; ${ }^{10}$ Present address: Kennedy Krieger Institute, Baltimore, MD, USA; ${ }^{11}$ Present address: LabCorp, Austin, TX, USA; ${ }^{12}$ Present address: Illumina, Houston, TX, USA. Correspondence: Lisa T. Emrick (emrick@bcm.edu) Members of the Undiagnosed Diseases Network are collaborators; complete list provided as a supplement.
} 
(UDN) with a known chromosomal deletion involving 17 p13.3 because the pathogenicity of the deletion and the etiology of her disease were not well established. Review of Baylor Genetics' cytogenetic database for individuals with similar deletions limited to the region between $Y W H A E / C R K$ and PAFAH1B1 identified three other individuals with white matter changes and one individual with a normal brain magnetic resonance image (MRI). Two of these individuals were brothers (subjects 2-3) and also enrolled in the UDN. Subject 5 was referred after an investigator (A.V.) learned learned of the study cohort. A literature search with 17 p13 search terms allowed identification of other reports of similar white matter changes in individuals with deletions involving this region. No individuals in the DECIPHER database with deletions in the same region showed similar white matter changes, although MRI information was only available for a single individual (2726). Informed consent was obtained to publish identifiable clinical data presented in this paper.

\section{Chromosomal microarray testing}

V8, V9, and V10 chromosomal microarrays (CMAs) were designed by Baylor Medical Genetics Laboratories and manufactured by Agilent (Santa Clara, CA, USA). Subjects 2,4 , and 6 were studied by the V8 array, which included approximately 180,000 oligonucleotides and covered $\sim 1714$ genes with an average of 4.2 probes per exon, excluding lowcopy repeats and other repetitive sequences. ${ }^{6}$ Subjects 3 and 1 were studied by the V9 and V10 arrays respectively, which targeted over 4900 genes at the exon level plus 60,000 probes used for single-nucleotide polymorphism (SNP) analysis for the detection of uniparental disomy and absence of heterozygosity. $^{7}$

\section{Exome sequencing}

Subjects 1 and 2 had exome sequencing according to previously described methods. ${ }^{8}$

\section{Genome sequencing}

Subject 5 had genome sequencing through the Illumina Clinical Services Laboratory TruGenome Undiagnosed Disease test. Sequencing libraries were prepared using Illumina's TruSeq DNA PCR-Free kit and sequenced on a HiSeqX. Variant calling was performed using the Illumina ISAS pipeline, with single-nucleotide variants (SNVs) and indels called using the Strelka germline caller ${ }^{9}$ and copy-number variants (CNVs) identified with Canvas. ${ }^{10}$ All variants were classified according to the American College of Medical Genetics and Genomics standards and guidelines for interpretation of genetic sequence variants.

\section{RESULTS}

Subject 1 was born full-term and required phototherapy for hyperbilirubinemia. She met all of her early milestones and attends regular classes but has difficulty with short-term memory.
At age 7 years she had recurrent vomiting after a viral illness. Additional symptoms included headaches, dizziness, inability to concentrate, and tingling in the legs. Brain and spine MRI showed significant white matter hyperintensities in the brain (Fig. 1a), a Chiari I malformation, and a tethered cord. The white matter lesions remained unchanged on subsequent MRIs. Additional anomalies include a patent ductus arteriosus, a low IgA screen for celiac disease, and joint laxity (Beighton score 4/9). On examination she is normocephalic and nondysmorphic. Genetic workup included normal metabolic testing for arylsulfatase A, galactocerebrosidase, and thymidine levels. Cerebrospinal fluid (CSF) analysis showed normal protein, glucose, and lactate. Infectious workup for tuberculosis, enterovirus and herpes simplex virus (HSV) was negative. Chromosomal microarray (CMA) showed a $0.7-\mathrm{Mb}$ de novo 17p13.3 deletion (Fig. 1e, Table 1). Exome sequencing did not show any pathogenic variants or candidate genes associated with white matter changes, or rare variants in the nondeleted $17 \mathrm{p} 13.3$ alleles.

Subject 2 was born at 33 weeks secondary to preterm labor. He was in the neonatal intensive care unit for 24 days and had a grade I intraventricular hemorrhage. He had early developmental delays but a normal IQ and attention deficit hyperactivity disorder (ADHD) at age 6. He underwent a brain MRI for worsening behaviors without regression that showed extensive bilateral multifocal T2 hyperintensities (Fig. 1b). Multiple subsequent brain MRIs were stable. Physical exam showed macrocephaly with head circumference $>95 \%$, frontal bossing, and hypermobile joints (Beighton score 6/9). Echocardiogram and eye exams were normal. Genetic workup included normal urine mucopolysaccharide screening, serum very long chain fatty acids, and arylsulfatase activity. CMA showed a $0.8-\mathrm{Mb} 17 \mathrm{p} 13.3$ deletion (Fig. 1e, Table 1) and a small deletion on $15 \mathrm{q} 11.2$ including only noncoding exons of SNRPN. The $17 \mathrm{p} 13.3$ deletion was inherited from his father and shared with a younger brother (individual 3). Their father may also be similarly affected. He had problems with rolling his ankles when he was younger, as well as behavior issues. He has a cranial metal plate after a motor vehicle accident and therefore is unable to obtain a brain MRI, but a computed tomography (CT) scan at the time of the accident showed old white matter changes; additional details are not available. In individual 2, exome sequencing did not show any pathogenic variants or candidate genes associated with white matter changes, or rare variants in the nondeleted $17 \mathrm{p} 13.3$ alleles.

Subject 3 was born full-term with normal delivery and newborn course. He met all of his early milestones. Brain MRI at 3 years, performed due to macrocephaly and his brother's MRI findings, showed patchy punctate bilateral multifocal T2 hyperintensities (Fig. 1c) and two small arachnoid cysts. Multiple subsequent brain MRIs were stable. His exam is significant for head circumference $>98 \%$ with frontal bossing and hypermobile joints (Beighton score 6/9). Echocardiogram showed mild mitral and tricuspid regurgitation. He was diagnosed with $\mathrm{ADHD}$ at age 5. 

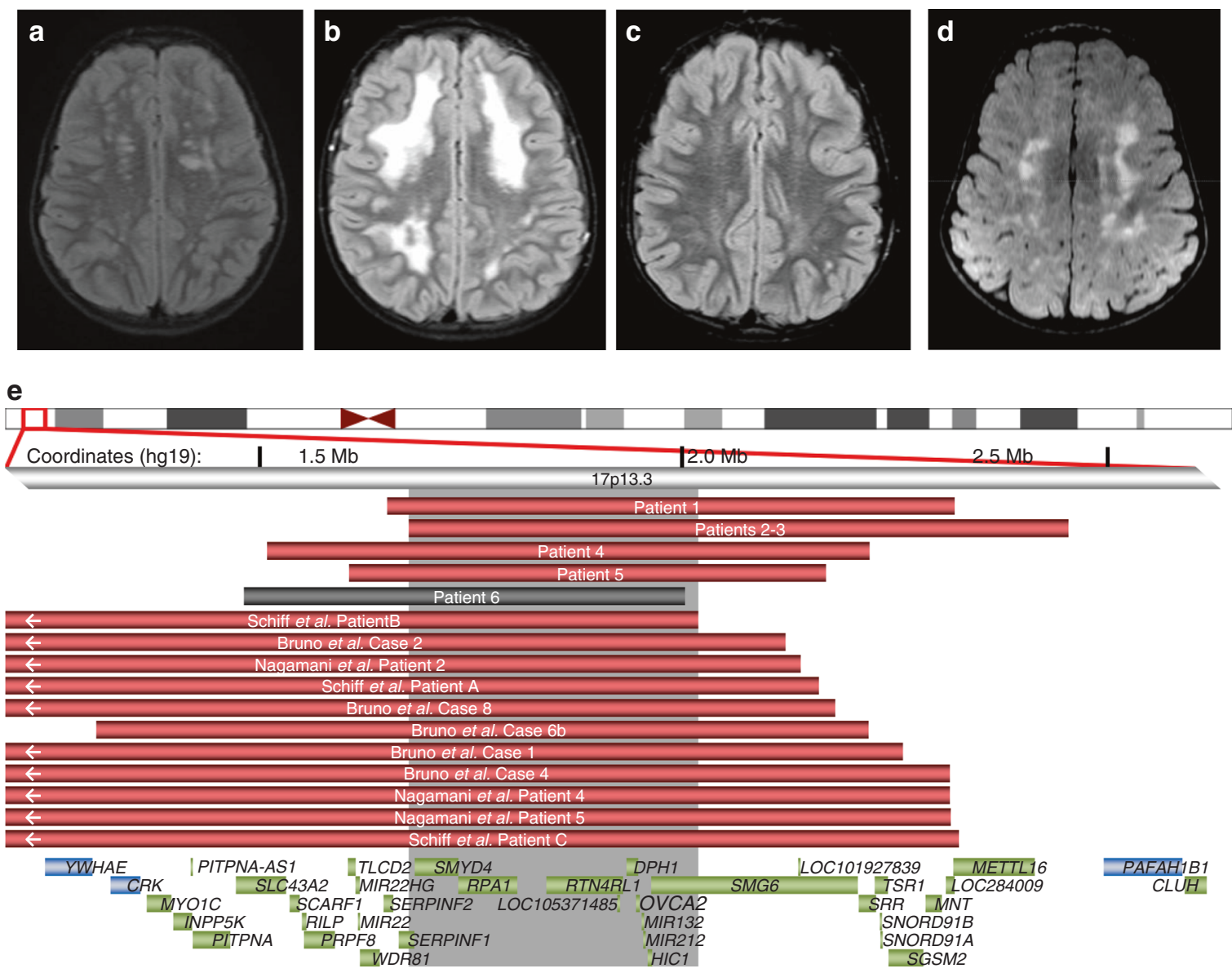

Fig. 1 Leukoencephalopathies in individuals with 17p13.3 deletions. Axial T2 weighted fluid attenuated inversion recovery (FLAIR) images demonstrate bilateral T2 hyperintensities in subject $1(\mathbf{a})$, subject $2(\mathbf{b})$, subject $3(\mathbf{c})$, and subject $5(\mathbf{d})$. (e) Deletions associated with abnormal magnetic resonance images (MRIs) in our cohort and review of the literature are red; the individual with a normal MRI is shown in gray. Gray shaded area represents the smallest region of overlap among cases with characteristic leukoencephalopathy. Genes in the region are shown as green boxes, with the critical genes of note flanking the region shown in blue.

Subject 4 is a 3 -year-old male with limited additional information secondary to loss of follow-up. MRI reported periventricular leukomalacia. CMA showed a $0.7-\mathrm{Mb}$ maternally inherited 17p13.3 deletion (Fig. 1e, Table 1).

Subject 5 was born full-term with no neonatal complications. Early motor and language development were normal. He underwent brain MRI due to low IGF-1 and growth hormone deficiency. He has a normal pituitary but incidentally found bilateral T2 hyperintensities in the brain (Fig. 1d). An extensive evaluation for leukodystrophy was unremarkable apart from a de novo $0.6-\mathrm{Mb} 17 \mathrm{p} 13.3$ deletion (Fig. 1d, Table 1) detected through research genome sequencing. At age 3 years, the individual has normal development and neurological exam.

Subject 6i was referred to genetics at 7 -years-old for short stature. She has relative microcephaly with dysmorphic features including deep-set eyes, upslanting palpebral fissures, mild midface hypoplasia, and a pointed chin. Normal laboratory workup included thyroid studies, urinalysis, and IGF-1. Brain MRI performed at 9 years did not show white matter abnormalities. CMA showed a $0.5-\mathrm{Mb}$ de novo 17p13.3 deletion (Fig. 1e, Table 1).

\section{DISCUSSION}

Microdeletion syndromes involving 17p13.3 have a range of phenotypes, with the most severe being Miller-Dieker syndrome, with primary lissencephaly, growth failure, intellectual disability, and dysmorphic features. ${ }^{1}$ Genotype-phenotype correlations have elucidated the roles of genes: PAFAH1B1 involved in brain development ${ }^{1}$ and $C R K$ in short stature. ${ }^{2,3}$ In reviewing the literature, similar white matter changes have also been reported in 11 other individuals with microdeletions in this region (Fig. 1e, Table S1). ${ }^{2-4}$ We report a small cohort of children with white matter lesions and normal cognition with small 17p13.3 microdeletions between and not including YWHAE/CRK and PAFAH1B1. Previous literature has speculated a possible role for YWHAE in the brain anomalies, including the white matter changes. ${ }^{2-5}$ Our cohort suggests a different gene is likely responsible for the white matter findings, although it is 


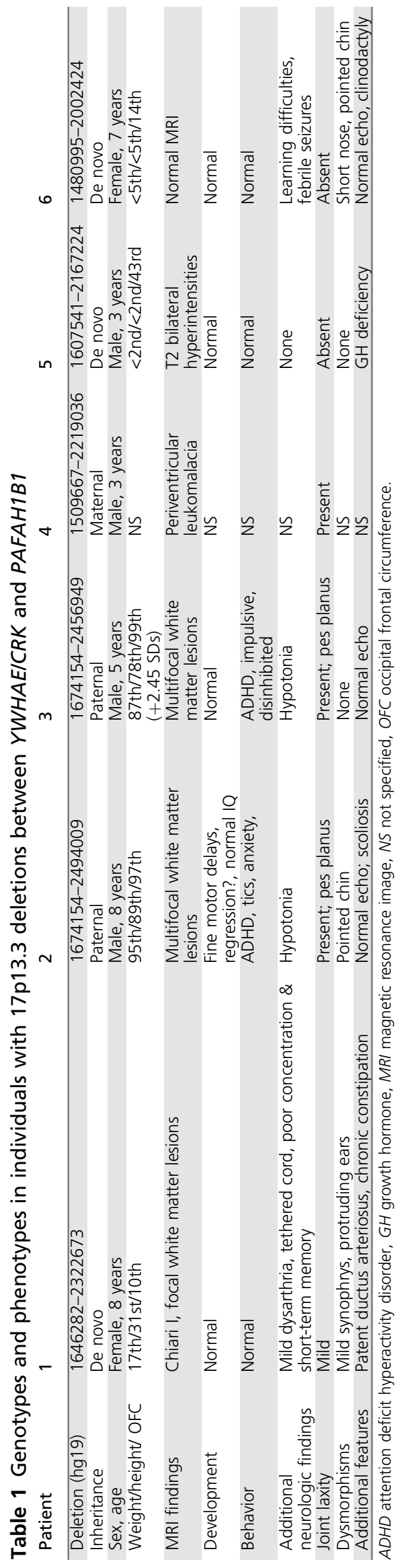

likely that multiple $17 \mathrm{p} 13.3$ genes contribute to brain development. Due to the normal MRI in individual 6 whose deletion includes all genes in the smallest region of overlap (SRO) for this phenotype (Fig. 1e), reduced penetrance is likely for these white matter changes.

White matter changes in the brain are often concerning for possible neurodegenerative leukodystrophy. Our cohort demonstrates that white matter changes can be associated with static, less severe conditions. The lesions in individuals 1-3 appear to be static based on multiple brain MRIs in each individual and the likely presence of similar findings in the father of individuals 2 and 3 . The white matter changes in our cohort and those previously reported who have MRI images to review are similar, located mostly in the subcortical regions suggestive of enlarged perivascular spaces. There is no evidence of more severe brain injury such as gliosis that may have been associated with prematurity or an infectious process.

There are 11 RefSeq genes within our newly defined SRO (Fig. 1e) for these white matter changes, and none has been previously associated with such brain findings. It is unclear whether individual genetic changes are responsible for white matter abnormalities in these individuals or if these are a feature of CNVs in general. Similar white matter changes have been described in individuals with other CNVs, although for the most part no causative genes have been confirmed within CNVs. ${ }^{11}$ Exceptions, for example, include haploinsufficiency of $M B P$ (myelin basic protein) in 18q23 deletions, which have been linked to white matter changes on MRI. ${ }^{12}$

Most of these genes found in our SRO are not associated with Mendelian disorders, although RTN4RL1, SMG6, MIR132, and MIR212 are expressed in the brain. SMG6 and RTN4RL1 have the highest probabilities of loss-of-function intolerance (pLIs, ${ }^{13} 1.00$ and 0.79 , respectively) of the SRO genes. RTN4RL1 regulates axonal and dendritic growth, ${ }^{14}$ and it may serve as a receptor for Nogo-66, a myelin-associated inhibitor. ${ }^{15}$ Of note, the Nogo-66 receptor gene RTN4R is located in 22q11.21, recurrent deletions of which can cause white matter abnormalities, a phenotype that has been hypothesized to be further modulated by polymorphisms in the nondeleted RTN4R allele. ${ }^{16}$ SMG6 is involved in messenger RNA (mRNA) decay. ${ }^{17}$ As microRNAs MIR132 and MIR212 are noncoding, pLI scores are not applicable, although attenuation of Mir132 in mice reduces neurite outgrowth. ${ }^{18}$

It has been hypothesized that HIC1 haploinsufficiency (pLI score not available) contributes to the facial features and heart and gastrointestinal anomalies in Miller-Dieker syndrome, ${ }^{19}$ although these features are not prominent in our cohort. Given HIC1's role in precartilaginous tissues and muscle development, ${ }^{19}$ this could be a candidate for the hypermobility in some of our individuals. Additionally, as HIC1 acts as a tumor suppressor, ${ }^{20}$ future monitoring of individuals with $\mathrm{HIC1}$ deletions for risk of neoplasia is possibly warranted. The remainder of the genes in the SRO have low pLI scores, and two (SERPINF1 and DPH1) are associated with recessive 
disease (OMIM 613982 and 616901), so it is less likely that these genes are haploinsufficient. However, as these white matter changes may have minimal outward phenotypic impacts, we cannot rule out the possibility that one of these other genes with more tolerance to loss of function contributes to the MRI findings.

When white matter changes are found, long-term follow-up is sometimes recommended to evaluate whether the leukoencephalopathy is static. Given the likely presence of such changes in an adult (father of individuals 2-3) and normal development in our cohort over time, reassurance may be given to families with similar 17p13.3 deletions regarding the likely evolution of their white matter changes over time, and repeated MRIs should not be mandatory.

Our cohort contributes evidence to the presence of multiple genes within 17p13.3 required for normal brain development. Identification of additional individuals with deletions and pathogenic variants within this region may help to identify specific genes responsible. Additional studies, including diffusion tensor imaging and downstream techniques such as RNAseq to evaluate for abnormal expression of genes within and adjacent to the deletion and in relevant developmental pathways, may assist with elucidating genetic etiologies of abnormal myelination.

\section{ACKNOWLEDGEMENTS}

We thank the individuals and their families for their participation in this research. Research reported in this manuscript was supported by the National Institutes of Health $(\mathrm{NIH})$ Common Fund, through the Office of Strategic Coordination/Office of the $\mathrm{NIH}$ Director under award number U01 HG007709-01. The content is solely the responsibility of the authors and does not necessarily represent the official views of the National Institutes of Health. This work was also supported by the Baylor College of Medicine Intellectual and Developmental Disabilities Research Center (HD024064) from the Eunice Kennedy Shriver National Institute of Child Health and National Institute of General Medical Sciences (NIGMS) T32 GM007526 (B.L., L.T.E., M.J., L.C.B., J.W.B.). L.C.B. is also supported by a Career Award for Medical Scientists from the Burroughs Wellcome Fund and NIH K08DK106453.

\section{DISCLOSURE}

The Department of Molecular and Human Genetics at Baylor College of Medicine receives revenue from clinical genetic testing conducted at Baylor Genetics, including chromosomal microarray testing. J.W.B., R.J.T., D.P., and K.B. are employees and shareholders of Illumina. H.N. is an employee of LabCorp. The other authors declare no conflicts of interest.

\section{REFERENCES}

1. Dobyns WB, Das S. LIS1-associated lissencephaly/subcortical band heterotopia. In: Adam MP, Ardinger HH, Pagon RAGeneReviews. Seattle, WA: University of Washington; 1993.

2. Nagamani SC, Zhang F, Shchelochkov OA, et al. Microdeletions including YWHAE in the Miller-Dieker syndrome region on chromosome 17p13.3 result in facial dysmorphisms, growth restriction, and cognitive impairment. J Med Genet. 2009;46:825-833.

3. Bruno DL, Anderlid BM, Lindstrand A, et al. Further molecular and clinical delineation of co-locating 17p13.3 microdeletions and microduplications that show distinctive phenotypes. J Med Genet. 2010;47:299-311.

4. Schiff $M$, Delahaye A, Andrieux J, et al. Further delineation of the 17p13.3 microdeletion involving YWHAE but distal to PAFAH1B1: four additional individuals. Eur J Med Genet. 2010;53:303-308.

5. Noor A, Bogatan S, Watkins N, Meschino WS, Stavropoulos DJ. Disruption of YWHAE gene at 17p13.3 causes learning disabilities and brain abnormalities. Clin Genet. 2018;93:365-367.

6. Chaudhry A, Noor A, Degagne B, et al. Phenotypic spectrum associated with PTCHD1 deletions and truncating mutations includes intellectual disability and autism spectrum disorder. Clin Genet. 2015;88:224-233.

7. Wiszniewska J, Bi W, Shaw C, et al. Combined array $\mathrm{CGH}$ plus SNP genome analyses in a single assay for optimized clinical testing. Eur J Hum Genet. 2014;22:79-87.

8. Yang $\mathrm{Y}$, Muzny DM, Xia F, et al. Molecular findings among individuals referred for clinical whole-exome sequencing. JAMA. 2014;312:1870-1879.

9. Saunders CT, Wong WS, Swamy S, Becq J, Murray LJ, Cheetham RK. Strelka: accurate somatic small-variant calling from sequenced tumornormal sample pairs. Bioinformatics. 2012;28:1811-1817.

10. Ivakhno S, Roller E, Colombo C, Tedder P, Cox AJ. Canvas SPW: calling de novo copy number variants in pedigrees. Bioinformatics. 2018; 516-518.

11. Garcia-Cazorla A, Sans A, Baquero M, et al. White matter alterations associated with chromosomal disorders. Dev Med Child Neurol. 2004;46:148-153.

12. Loevner LA, Shapiro RM, Grossman RI, Overhauser J, Kamholz J. White matter changes associated with deletions of the long arm of chromosome 18 (18q- syndrome): a dysmyelinating disorder? AJNR Am J Neuroradiol. 1996;17:1843-1848.

13. Lek M, Karczewski KJ, Minikel EV, et al. Analysis of protein-coding genetic variation in 60,706 humans. Nature. 2016;536:285-291.

14. Pignot $V$, Hein $A E$, Barske $C$, et al. Characterization of two novel proteins, $\mathrm{NgRH} 1$ and $\mathrm{NgRH} 2$, structurally and biochemically homologous to the Nogo-66 receptor. J Neurochem. 2003;85:717-728.

15. Zhang L, Kuang $X$, Zhang J. Nogo receptor 3, a paralog of Nogo-66 receptor 1 (NgR1), may function as a NgR1 co-receptor for Nogo-66. J Genet genomics. 2011;38:515-523.

16. Perlstein MD, Chohan MR, Coman IL, et al. White matter abnormalities in 22q11.2 deletion syndrome: preliminary associations with the Nogo-66 receptor gene and symptoms of psychosis. Schizophr Res. 2014;152:117-123.

17. Ottens F, Boehm V, Sibley CR, Ule J, Gehring NH. Transcript-specific characteristics determine the contribution of endo- and exonucleolytic decay pathways during the degradation of nonsense-mediated decay substrates. RNA. 2017;23:1224-1236.

18. Vo $\mathrm{N}$, Klein ME, Varlamova $\mathrm{O}$, et al. A cAMP-response element binding protein-induced microRNA regulates neuronal morphogenesis. Proc Natl Acad Sci USA. 2005;102:16426-16431.

19. Grimm C, Sporle R, Schmid TE, et al. Isolation and embryonic expression of the novel mouse gene Hic1, the homologue of HIC1, a candidate gene for the Miller-Dieker syndrome. Hum Mol Genet. 1999;8:697-710.

20. Fleuriel C, Touka M, Boulay G, Guerardel C, Rood BR, Leprince D. HIC1 (hypermethylated in cancer 1) epigenetic silencing in tumors. Int J Biochem Cell Biol. 2009;41:26-33. 